\title{
Dropsy and pyrexia of unknown origin: Tuberculosis myopericarditis, still the great pretender
}

\author{
Authors: Shilpa Manupati, ${ }^{A}$ Fozia Ahmed ${ }^{B}$ and David Shelton ${ }^{B}$
}

Tuberculosis is an infectious disease with a global presence. ${ }^{1}$ Primary myopericardial involvement presenting with congestive cardiac failure and pyrexia of unknown origin is extremely rare. ${ }^{2}$

A 30-year-old, male Pakistani construction worker presented with a 2-month history of worsening shortness of breath and peripheral oedema. He had not undertaken any recent foreign travel and reported non-specific, self-limiting flu-like symptoms approximately 1-month prior to the onset of his presenting symptoms. He had no cardiovascular risk factors or any positive relevant family history. Initial examination showed features of congestive cardiac failure with no evidence of active infection.

Trans-thoracic echocardiogram demonstrated left ventricular (LV) ejection fraction (LVEF) $<35 \%$ and a global pericardial effusion with no features of cardiac tamponade. He was treated for congestive cardiac failure.

Two weeks into the hospitalisation he became pyrexial. Extensive bacterial, fungal, viral and HIV screening, and screening for vasculitis and autoimmune serology (including rheumatoid factor and anti-neutrophil cytoplasm antibodies), were unremarkable. Multiple early morning sputum samples were negative for acid-fast bacillus (AFB) with no growth on cultures.

Ultrasound-guided diagnostic pleural tap revealed an exudative effusion, negative for infections including AFB and malignant cells. Computed tomography (CT) of thorax, abdomen and pelvis was undertaken to identify the primary source of occult infection. This demonstrated bilateral supraclavicular lymphadenopathy and massive right pleural effusion causing midline shift. Pleural drainage yielded over 3 litres of straw-coloured fluid. Analysis once again confirmed an exudative effusion with lymphocytes, no AFB or evidence of malignancy.

Repeat contrast CT showed significant mediastinal and hilar lymphadenopathy, moderate pericardial effusion, and a recurrence of the right pleural effusion with no abdominal or pelvic lymphadenopathy.

After 13 days of empirical antituberculosis treatment (ATT), endoscopic bronchial ultrasound with fine needle aspiration cytology from the lower paratracheal nodes confirmed granulomatous lymphadenitis with no malignant cells. Cultures for AFB remained negative.

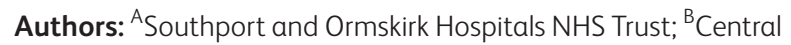
Manchester NHS Foundation Trust
He was treated with multiple courses of antibiotics for presumed hospital-acquired pneumonia when there was initial onset of pyrexia with respiratory findings. He continued to spike temperatures, and blood markers for infection remained elevated. After exclusion of lymphoma, the patient was commenced on quadruple ATT with a reducing course of oral prednisolone for presumed pleural, pericardial and lymph node tuberculosis under the guidance of respiratory and infectious diseases team.

Within a week of starting ATT, there was a rapid decline in pyrexia, infection markers and features of heart failure. Serial echocardiography demonstrated a temporal improvement in LVEF. Follow up was arranged in the heart failure and respiratory clinics.

\section{Learning points}

> Cardiac tuberculosis is a potentially treatable and reversible cause of cardiac failure.

> Always consider a diagnosis of myocardial tuberculosis in patients with refractory ventricular failure or uncontrollable ventricular arrhythmias in patients at risk of current of historical tuberculosis exposures without any cardiac risk factors.

> Commence treatment based on clinical suspicion and be guided by the patient response, even in the absence of definitive tissue diagnosis of tuberculosis.

\section{Conflict of interest statement}

None declared.

\section{References}

1 World Health Organization. Tuberculosis. Geneva: WHO, 2019. www. who.int/mediacentre/factsheets/fs104/en/ [Accessed 27 February 2019].

2 Michira B, Alkizim F, Matheka D. Patterns and clinical manifestations of tuberculous myocarditis: a systematic review of cases. Pan Afr Med J 2015;21:118. 